\title{
AVALIAÇÃO MORFOLÓGICA E NUCLEAR DE OÓCITOS BOVINOS IMATUROS, OBTIDOS DE OVÁRIOS COM E SEM A PRESENÇA DE CORPO LÚTEO
}

Juliana Ferreira Batista, Leandro Francisco da Silva, Leandro Polato Lazari, Mariana das Graças de Oliveira Leal, Matheus Montanari de Souza, Sheila Merlo Garcia.

Universidade do Oeste Paulista -UNOESTE, Curso de Zootecnia, Presidente Prudente, SP. e-mail: julianafb93@hotmail.com

\section{RESUMO}

A produção in vitro de embriões (PIVE) é uma biotecnologia que possibilita principalmente o rápido aumento no número de descendentes com mérito genético superior. Atualmente o Brasil é líder mundial na PIVE e grande parte desse sucesso deve-se às inúmeras pesquisas realizadas nessa área. A seleção de oócitos de boa qualidade para essa técnica é determinante para obtenção de embriões de qualidade e satisfatórios índices de prenhez. Desta forma, esse trabalho teve como objetivo avaliar oócitos, obtidos de ovários com e sem a presença de corpo lúteo $(\mathrm{CL})$, de acordo com a classificação morfológica e nuclear, afim de identificar a possível influência do CL na viabilidade dos oócitos. Foram coletados ovários de abatedouro e separados em dois grupos, com e sem CL. Os folículos foram aspirados, e os oócitos obtidos foram selecionados, classificados e em seguida corados com Hoechst 33342 e avaliados sob microscópio de epifluorescência, para avaliação nuclear. Realizou-se análise descritiva de cada tratamento com relação a classificação morfológica e nuclear. Os resultados obtidos foram analisados através de testes não-paramétricos, sendo o efeito do corpo lúteo verificado por meio do teste Quiquadrado $\left(\chi^{2}\right)$ ( $R$ core Team, 2013). Concluiu-se que a presença de $C L$ não influencia na qualidade de oócitos imaturos, do ponto de vista quantitativo, morfológico e nuclear.

Palavras-chave: complexo Cumulus-Oócito; PIVE; meiose.

\section{MORPHOLOGICAL EVALUATION AND NUCLEAR OOCYTES IMMATURE, OBTAINED FROM OVARIAN WITH AND WITHOUT THE BODY PRESENCE LUTEUM}

\begin{abstract}
The in vitro embryo production (IVP) is a biotechnology that primarily enables the rapid increase in the number of offspring with superior genetic merit . Currently, Brazil is the world leader in IVP and much of this success is due to the extensive research carried out in that area. The selection of good quality oocytes for this technique is critical to obtaining quality embryos and satisfactory pregnancy rates. Thus, this study aimed to assess oocytes obtained from ovaries with and without the presence of corpus luteum (CL), according to the morphological and nuclear classification, in order to identify the possible influence of $\mathrm{CL}$ on the viability of oocytes. They were collected from slaughterhouse ovaries and separated into two groups, with and without CL. Follicles were aspirated and the oocytes were selected, ranked, and then stained with Hoechst 33342 and evaluated by epifluorescence microscope for nuclear evaluation. A descriptive analysis of each treatment with respect to morphological and nuclear classification. The results were analyzed using non-parametric tests, with the effect of the corpus luteum verified using the chisquare test ( $\chi 2$ ) ( $R$ Core Team, 2013). It was concluded that the presence of $C L$ does not influence the quality of immature oocytes, from the quantitative and morphological nuclear standpoint.
\end{abstract}

Keywords: Cumulus-Oocyte complex; IVP; meiosis.

\section{INTRODUÇÃO}

O Brasil é o maior produtor de embriões in vitro (PIVE) do mundo com uma produção em torno de 320 mil embriões por ano, o que representa $50 \%$ do mercado mundial (BRASIL, 2016), e confere ao país considerável destaque na bovinocultura. A PIVE é uma biotecnologia que aumenta o número de descendentes com mérito 
genético superior e seu sucesso deve-se graças as pesquisas na área de reprodução animal, principalmente aquelas que buscam melhorar a qualidade dos oócitos para completar todas as etapas da técnica (GONÇALVES et al., 2008).

A PIVE possui três etapas para que os embriões possam ser transferidos ou criopreservados: a maturação in vitro (MIV), fertilização in vitro (FIV) e cultivo in vitro (CIV) (VARAGO et al., 2008).

Segundo Velho (2011), essa é uma tecnologia onde o principal desafio é aumentar a porcentagem de embriões que alcançam os estádios de blastocisto, e que apesar das etapas anteriores ocorrerem de forma normal, essa taxa dificilmente é superior a $40 \%$. Evidências indicam que a qualidade e competência do oócito é fundamental para desenvolver embriões de boa qualidade.

Vários fatores influenciam na baixa taxa de produção de embriões in vitro, quando comparada com a produção in vivo, como por exemplo, a retirada do oócito do folículo, fatores ambientais externos ou ainda falhas em alguma das etapas da PIVE (maturação, fecundação, cultivo) (GOTTARDI; MINGOTI, 2009).

A fase responsável por preparar os oócitos de forma artificial para a fecundação e posterior desenvolvimento embrionário é a MIV (GONÇALVES et al., 2008). Nessa etapa, os oócitos que estão em prófase $\mathrm{I}$, retomam a meiose e atingem a metáfase II, ficando prontos para serem fecundados (SMITZ et al., 2004). Assim, a seleção de oócitos de boa qualidade é fundamental para garantir o sucesso de todas as etapas, garantindo uma maior taxa de embriões.

Esse processo pode ser feito por meio de oócitos obtidos de ovários oriundos de abatedouro, onde são muitas as variáveis que interferem na sua qualidade, sendo a presença do corpo lúteo $(\mathrm{CL})$ uma delas.

$\mathrm{O} C \mathrm{~L}$ é uma glândula endócrina formada no ovário após uma ovulação, e contribui para a regulação do ciclo estral e manutenção da gestação. Sua principal função é secretar progesterona (P4) durante o ciclo estral, bem como durante a gestação (BARBOSA et al., 2013).

Esse trabalho teve como objetivo avaliar a quantidade e qualidade de oócitos obtidos de ovários com e sem a presença de $\mathrm{CL}$, oriundos de abatedouro, de acordo com a classificação morfológica e a fase nuclear da meiose, afim de identificar a possível influência do $\mathrm{CL}$, na viabilidade dos oócitos para MIV.

\section{METODOLOGIA}

O estudo foi desenvolvido no laboratório de PIVE do mestrado em Ciência Animal da Universidade do Oeste Paulista - UNOESTE Campus II - Presidente Prudente - SP. Para a realização do experimento foram utilizados ovários de fêmeas bovinas, provenientes do abatedouro Bom Marte, localizado no município de Presidente Prudente - SP. Os ovários bovinos foram transportados para o laboratório em garrafa térmica contendo solução salina a 0,9\% de $\mathrm{NaCl}$, na temperatura de $38^{\circ} \mathrm{C}$, em um período máximo de uma hora.

No laboratório os ovários, foram lavados com solução salina e mantidos em banho-maria a $38^{\circ} \mathrm{C}$ até a aspiração. Antes da aspiração foram separados em dois grupos, G1: com a presença de $C L$ e G2: sem a presença de CL. Para a aspiração folicular os ovários foram limpos com gaze estéril, e os oócitos foram aspirados com auxílio de seringas de $20 \mathrm{~mL}$ com extremidade de agulha 18G. Após a aspiração o líquido folicular foi transferido para tubos de ensaio mantidos em banho-maria a $38^{\circ} \mathrm{C}$ e decantado para separação dos oócitos. Após desprezar-se o sobrenadante, o sedimento foi depositado em placa de petrimarcada, para posterior procura e seleção dos oócitos, em lupa esteromicroscópica (Forty, American Optical Corporation, com aumento de 4,5x).

\section{Os oócitos recuperados}

foram classificados de acordo com $\mathrm{o}$ aspecto morfológico dos complexo cumulus-

oócitos (COC's), segundo Lonergan (1992), em 5 grupos de qualidade, onde Grau 1 = células do cumulus compacto presente, contendo mais de três camadas de células; Grau II = células do cumulus compacto parcialmente presente em volta do oócito ou rodeando completamente o oócito, com menos de três camadas celulares; Grau III = células do cumulus presente, com apenas uma camada de célula; Desnudo = ausência de camada de células do cumulus e Atrésicos = células do cumulus em regressão celular. A seguir, somente os oócitos classificados em grau I, II e III, foram selecionados.

Com o intuito de observar a qualidade dos oócitos selecionados avaliou-se o estádio nuclear que esses encontravam-se antes de 
serem submetidos à maturação. Primeiramente, para a remoção das células do cumulus, os oócitos foram depositados em ependorf contendo hialuronidase $0,2 \%$ (Hyalozima ${ }^{\circledR}$, Aspen) e desnudados com vórtex, velocidade 6 por 5 minutos. Posteriormente, os oócitos foram lavados em solução de bloqueio(SB),[PBS com $1 \mathrm{mg} / \mathrm{mL}$ de albumina sérica bovina(BSA; SigmaA-6003)] e fixados em $500 \mu \mathrm{L}$ de paraformaldeído $4 \%$ por 30 minutos à temperatura ambiente, em placa de quatro poços. Após esse tempo foram lavados três vezes em SB, e permeabilizados por 15 minutos a $38^{\circ} \mathrm{C}$ em SB acrescida de $0,1 \%$ de Triton X- 100. Os oócitos foram então lavados três vezes em SB, e corados com $10 \mu \mathrm{g} / \mathrm{mL}$ de Hoechst 33342 em PBS por 10 minutos.

Após o processo de coloração os oócitos foram transferidos para lâminas de vidro, em gotas $10 \mu \mathrm{L}$ de glicerol, recobertas com lamínulas e vedadas com esmalte. As lâminas foram analisadas no laboratório da Agência Paulista de Tecnologia dos Agronegócios (APTA), em Presidente Prudente-SP, situado na Rodovia Raposo Tavares, km 561.

Os oócitos foram avaliados quanto a fase nuclear em microscópio de epifluorescência (Olympus - IX-51, objetiva de 40x, excitação 330-385nm e emissão 420-490nm para o Hoechst).

As fases nucleares dos oócitos imaturos foram avaliadas segundo Woudenberg et al. (2012), por graus variados de condensação dos cromossomos, como segue: A- Vesícula germinativa (VG, estádio dictiato da prófase I) Presença de núcleo vesicular, envelope nuclear e cromossomos apresentando-se pouco condensados; B - Quebra da Vesícula Germinativa (QVG) - Cromossomos com pequeno grau de condensação e dispersa distribuição, porém ainda com núcleo de aspecto vesicular, mas sem a presença do envelope nuclear; $C$ Metáfase I (MI) - Cromossomos com grau mais avançado de condensação, não sendo possível sua visualização individual; D - Degenerados/Não passíveis de determinação (D/NI) - Oócitos cujo estádio de desenvolvimento nuclear não era passível de determinação ou não apresentavam cromatina evidente.

Os dados foram analisados através de testes não-paramétricos, sendo o efeito do corpo lúteo verificado por meio do teste Qui-quadrado (X2) (R CORE TEAM, 2013). Foram realizadas análise descritiva de cada tratamento, onde se observa a média, variância, desvio padrão e coeficiente de variância com relação a classificação morfológica e nuclear. O nível de significância utilizado para rejeitar HO (hipótese de nulidade) foi de $5 \%$, isto é, para $p<0,05$, considerou-se que não ocorreram diferenças significativas entre as variáveis.

\section{RESULTADOS}

Durante o trabalho foram utilizados no total, entre os dois tratamentos, 159 ovários e 536 oócitos, (76 grau I, 139 grau II, 222 grau III, 63 desnudos e 36 atrésicos) distribuídos em 4 repetições. Na primeira repetição, foram colhidos 37 ovários, selecionando-se 112 oócitos; na segunda repetição, obtiveram-se 40 ovários, sendo selecionados 145 oócitos; na terceira repetição o número de ovários foi de 18 , selecionando-se 105 oócitos, na quarta repetição o número de ovário foi de 64 e 177 oócitos.

Os resultados do índice de recuperação de oócitos de acordo com a classificação morfológica dos COC's dos dois grupos são mostrados na Tabela 1, onde observou-se que os grupos com $\mathrm{CL}$ e sem $\mathrm{CL}$ apresentaram $85 \%$ e $82 \%$ de oócitos viáveis respectivamente (grau I, grau II e grau III). No entanto, o número de oócitos totais aspirados em relação à porcentagem de oócitos viáveis e a média do oócito/ovário não deferiu entre os dois grupos.

Tabela 1. Índice de recuperação e classificação morfológica de oócitos imaturos, de ovários com e sem a presença de CL.

\begin{tabular}{|c|c|c|c|c|c|c|c|}
\hline \multirow{2}{*}{ Tratamento } & \multirow{2}{*}{$\begin{array}{l}\mathrm{N}^{\circ} \text { oócitos } \\
\text { aspirados }\end{array}$} & \multirow{2}{*}{$\begin{array}{c}\text { média } \\
\text { oócito/ovário }\end{array}$} & \multicolumn{5}{|c|}{ Classificação Morfológica } \\
\hline & & & n Grau I & n Grau II & n Grau III & n Desnudo & n Atrésico \\
\hline com CL & 262 & 3,54 & $15 \%$ & $27 \%$ & $43 \%$ & $10 \%$ & $6 \%$ \\
\hline sem CL & 277 & 3,26 & $14 \%$ & $26 \%$ & $42 \%$ & $15 \%$ & $9 \%$ \\
\hline
\end{tabular}

Fonte: Resultado da pesquisa. 
Com relação à avaliação do estádio nuclear do total de oócitos selecionados, 140 oócitos foram avaliados quanto ao estádio nuclear, sendo 63 oócitos do grupo de ovários com CL e 77 oócitos de ovários sem a presença de corpo lúteo. $\mathrm{Na}$ tabela 2 observa-se as porcentagens das fases nuclear de oócitos imaturos, de ovários com e sem a presença de $\mathrm{CL}$, onde não houve diferença entre os grupos.

Tabela 2. Diferenças percentuais das fases nuclear de oócito imaturos, de ovários com e sem a presença de CL.

\begin{tabular}{|l|c|c|c|c|c|}
\hline \multirow{2}{*}{ Tratamento } & \multirow{2}{*}{$\mathrm{N}^{\circ}$ oócitos } & \multicolumn{3}{|c|}{ Fases Nuclear } \\
\cline { 3 - 7 } & classificados & V G & Q V G & M I & Deg. \\
\hline com CL & 63 & $68 \%$ & $13 \%$ & $0 \%$ & $19 \%$ \\
\hline sem CL & 77 & $73 \%$ & $22 \%$ & $8 \%$ & $19 \%$ \\
\hline
\end{tabular}

Fonte: Resultado da pesquisa.

\section{DISCUSSÃO}

Neste trabalho a presença do $\mathrm{CL}$ não influenciou nos resultados. $O$ mesmo resultado foi observado por Sugulle et al. (2008), que investigou as taxas de clivagem e o desenvolvimento de blastocistos bovinos produzidos in vitro, de ovários com e sem CL. Dominguez (1995), relatou que a presença de CL, seja comparando animais com e sem $\mathrm{CL}$, ou no próprio animal (ovário com vs sem $\mathrm{CL}$ ), não afeta o número de folículos a serem aspirados nos ovários.

Existem vários fatores que influenciam na coleta dos oócitos, os quais são fundamentais para a maximização da taxa de recuperação dos mesmos. O grau de qualidade e a quantidade dos oócitos podem ser alterados pela raça, idade, estádio reprodutivo, condições climáticas, características foliculares, e condições experimentais - que envolvem todo o processo de retirada e transporte dos ovários até a aspiração folicular, incluindo a habilidade técnica e o tempo que leva todo esse processo. No entanto, os dados da literatura ainda são controversos com relação a influência do $\mathrm{CL}$ na quantidade e qualidade de oócitos.

O trabalho de Dode et al. (2000), ao analisar o número e o estádio nuclear de oócitos obtidos de diferentes tamanhos de folículos antes da maturação in vitro de vacas nelores, observou que mais de $80 \%$ de oócitos, independente da categoria folicular, encontra-se em estádio de vesícula germinativa, o que identifica oócitos imaturos.

Resultados semelhantes foram observados nesse trabalho, quando a maioria dos oócitos está em vesícula germinativa, o que era esperado, pelo fato de serem oócitos imaturos, além de confirmar a avaliação morfológica com relação à integridade do núcleo.

\section{CONCLUSÃO}

Nas condições experimentais a presença de $\mathrm{CL}$ não influencia na qualidade de oócitos imaturos, do ponto de vista morfológico e nuclear.

\section{REFERÊNCIAS}

BARBOSA, C.P.; TONIOLLO, G.H; GUIMARÃES, E.C.In vitro production of Nelore bovine embryos originated from oocytes from ovaries with and without corpus luteum. Ciência Animal Brasileira, v. 14, n. 1, p. 81-90, 2013.

BRASIL. Ministério da Agricultura, Pecuária e Abastecimento. Brasil está apto a exportar embriões bovinos in vitro para o Paraguai. Noticia, 2016. Disponível em: http://www.agricultura.gov.br/animal/noticias/2 016/08/brasil-esta-apto-a-exportar-embrioesbovinos-in-vitro-para-o-paraguai. Acesso em: 11 ago 2016.

DODE, M.A.N. et al. Effect of follicle size in nuclear and cytoplasmic maturation of oocytes from zebu cows. Pesquisa Agropecuária Brasileira, v. 35, n. 1, p. 207-214, 2000. https://doi.org/10.1590/S0100-

204X2000000100023

DOMINGUEZ, M.M. Effects of body condition, reproductive status and breed on follicular population and oocyte quality in cows. Theriogenology, v. 43 , n. 8, p. 1405-1418, Jul. $1995 . \quad$ https://doi.org/10.1016/0093691X(95)00126-S

GONÇALVES, P.B.D.; OLIVEIRA, M.A.L.; MEZZALIRA, A.; MONTAGNER, M.M.; VISITIN, J.A.; COSTA, L.F.S. Produção in vitro de embriões: 
Biotécnicas aplicadas à reprodução animal. 2.ed. São Paulo: Roca, 2008. p.261-291.

GOTTARDI, F. P.; MINGOTI, G. Z. Maturação de oócitos bovinos e influência na aquisição da competência para o desenvolvimento do embrião. Rev Bras Reprod Anim, v. 33, n. 2, p. 82-94, 2009.

LONERGAN, P. Studies in the in vitro maturation, fertilization and cultire of bovine follicular oocytes. 1992. PHD Thesis, National University of Ireland, Dublin.

R CORE TEAM. R. Na Introduction to R. Notes on R: A programming Environment for Data Analysis and Graphics. 2013. Available at: <http://crab.rproject.org/>.

SMITZ, J. E. J.; NOGUEIRA, D.; VANHOUTTE MATOS, D .G.; CORTVRINDT, R. N. Oocyte: in vitro maturation. In: Suh CS, Sonntag B, Erickson GF. The ovarian life cycle: a contemporany view. Rev Endocr Metab Disord, v. 3, p. 5-12, 2004.

SUGULLE, A.H.; DOCHI, O.; KOYAMA, H. Developmental competence of bovine oocytes selected by Brilliant Cresyl Blue Staining: Effect of the presence of corpus luteum on embryo development. Journal of Mammalian Ova Research, v. 25, n. 1, p. 50-55, Maio 2008. https://doi.org/10.1274/jmor.25.50

VARAGO, F.C; MENDONÇA, L.F; LAGARES, M.A. Produção in vitro de embriões bovinos: estado da arte e perspectiva de uma técnica em constante evolução. Rev. Bras. Reprod. Anim., Belo Horizonte, v.32, n.2, p.100-109, abr./jun. 2008.

VELHO, F.A.B. Avaliação da expressão gênica e da maturação nuclear in vitro em complexos cumuli-oócitos bovinos. 2011. 43f. Dissertação (Mestrado) - Faculdade de Veterinária, Universidade Federal do Rio Grande do Sul, Porto Alegre. Disponível em: <http://www.lume.ufrgs.br/handle/10183/34778 >. Acesso em: 10 out 2015.

WOUDENBERG, A.B.V. et al. The bovine oocyte in vitro maturation model: $A$ potential tool for reproductive toxicology screening. Reproductive Toxicology, v.34, p.251-260, 2012. Disponível em:

$<$ https://www.researchgate.net/publication/225 185510_The_bovine_oocyte_in_vitro_maturation _model_A_potential_tool_for_reproductive_toxi cology_screening $>$. Acesso em: 12 nov 2015.
Recebido para publicação em 16/08/2016

Revisado em 19/08/2016

Aceito em 26/08/2016 